\title{
Vehicle-to-Building is Economically Viable in Regulated Electricity Markets
}

\author{
Christian Gagné, Kevin Tanguy, and Karol Lina Lopez \\ Computer Vision and Systems Laboratory \\ Département de génie électrique et de génie informatique \\ Université Laval, Québec, QC, Canada G1V 0A6 \\ Emails: christian.gagne@ulaval.ca, \\ kevin.tanguy.1@ulaval.ca, karol-lina.lopez.1@ulaval.ca
}

\author{
Maxime R. Dubois \\ Département de génie électrique et de génie informatique \\ 2500 Boulevard de l'Université \\ Université de Sherbrooke, Sherbrooke, QC, Canada J1K 2R1 \\ Email: maxime.dubois@usherbrooke.ca.
}

\begin{abstract}
Vehicle-to-building (V2B) aims at exploiting electric vehicles (EVs) as occasional energy sources for smart buildings. In this paper, we are demonstrating the economic viability of V2B in the context of regulated electricity markets, taking into account the battery wear-out cost and charger inefficiencies. This is made possible thanks to the billing scheme used, which includes strong penalties for power calls exceeding subscribed capacities. Through the optimization of a realistic charging model, our simulations show that a fleet of hundreds of EVs can be charged for free, providing a significant benefit to the owners, while reducing the building's electricity bill.
\end{abstract}

Keywords-Vehicles-to-Building, Vehicles-to-Grid, Vehicle Electrification, Linear Programming

\section{INTRODUCTION}

Vehicle-to-grid (V2G) [1] consists in sporadically using energy stored in Electric Vehicles (EVs) to support the power grid. Such an innovative use of EVs has the potential of making the grid smarter, by exploiting energy sources located close to the consumers, while providing EV owners with some advantages (e.g., dividends lowering the overall EV ownership cost) for giving away some energy to the grid from time to time. However, although the principles of $\mathrm{V} 2 \mathrm{G}$ are appealing, the economic viability of the approach is not obvious in many circumstances.

Currently, one of the main obstacles to the approach is the battery wear-out cost induced by a V2G transaction. Indeed, using a value of $240 \$ / \mathrm{kWh}$ for standard Li-ion batteries and an expected useful life of 1500 cycles from $100 \%$ to $20 \%$ stateof-charge (SoC), it was estimated that the battery wear-out cost of $1 \mathrm{kWh}$ exchanged for $\mathrm{V} 2 \mathrm{G}$ is around $0.20 \$$, not taking into account the losses occurring in the charging process. Even with a more optimistic hypothesis, that means that the gains associated with V2G should be significant, given that the value of $1 \mathrm{kWh}$ obtained by $\mathrm{V} 2 \mathrm{G}$ is much higher than the average cost of $1 \mathrm{kWh}$ obtained from the power grid (typically around $0.10 \$$ in Canada and $0.12 \$$ in the US).

Intuitively, that would mean that for Canadian provinces such as Québec, Manitoba or British Columbia, where electricity is abundant and cheap, thanks to plentiful hydroelectric resources, and where the energy market is strongly regulated, with a fixed price over the day for consumers, the economic case for $\mathrm{V} 2 \mathrm{G}$ is hard to make. However, this reasoning does not take into account the fact that rates for medium and large consumers in such regulated markets are usually divided into two components, that is the amount of energy consumed and the maximum power call peak achieved during a period of time. These rates aim at maintaining the global power consumption at relatively constant level over time, in order to keep it within the production capacity of the grid.

In this paper, we are demonstrating that $\mathrm{V} 2 \mathrm{G}$ can be economically viable in a regulated electricity market. This is done through optimizing bidirectional charging of a small fleet of EVs (few 100s) on a university campus in Québec. Such an approach is an instance of vehicle-to-building (V2B) [2], where a form of demand-side management is made possible by exploiting the energy available in EVs for shaving power peaks of the buildings, allowing the power call over a month to be maintained within the subscribed power values. Given the significant penalties of the rate given to exceeding power calls at which the campus is bounded (i.e., "L" rate of HydroQuébec), our work is demonstrating that V2B is economic viability in the context of regulated electricity markets.

The remainder of the paper includes the following. First, the billing scheme and general system model is presented in Sec. II. Then, an overview of the optimization procedure is given in Sec. III, before detailing the experimental methodology of our experiments in Sec. IV. The results and analysis follow in Sec. V, and the conclusion is provided in Sec. VI.

\section{System MOdel}

This study will highlight the benefits of V2B when vehicles are to be connected to a parking or building infrastructure constrained with a fixed subscribed power rating. The specific example of the authors' campus of Université Laval is used for the remainder of the paper. Hydro-Québec, the provincial electricity provider, has a specific billing scheme for large power business customers (the so-called "L" rate) involving the following in two components:

- the maximum value of either 1) the subscribed power or 2) the maximum power peak in $\mathrm{kW}$ in the month;

- the total energy consumption in $\mathrm{kWh}$ during the month.

The power component is an important part of the bill, accounting for approximately $40 \%$ of the total cost. The specificity 
of this billing scheme involves the energy sold at a cheap and fixed price, depending on the season: $2.97 \phi$ per $\mathrm{kWh}$ in summer and $2.99 \varnothing$ per $\mathrm{kWh}$ in winter for the year 2011, while households pay $5.39 \notin$ per $\mathrm{kWh}$ at all times. The cost of power is calculated in several steps. First the power value to be billed is determined by retaining the highest value in $\mathrm{kW}$ between the maximum real power demand, $95 \%$ of the apparent power demand, and the subscribed power. The raw price of power is then calculated by multiplying this power value by $12.18 \$ / \mathrm{kW}$ and multiplying it again by the number of hours in the month divided by the number of hours in 30 days. However, the final cost of the power component takes into account two additional factors: a credit for supply at medium or high voltage $(0.915 \$ / \mathrm{kW})$ and an adjustment for transformation loss $(0.1670 \$ / \mathrm{kW}$ in summer, $0.16230 \$ / \mathrm{kW}$ in winter). In winter, the client must have an accurate rough estimate of his maximum power peak because if the value is over $110 \%$ of the subscribed power, a $7.11 \$$ daily penalty is applied per excessive $\mathrm{kW}$ (limited to $21.33 \$$ per excess $\mathrm{kW}$ monthly), in addition to the regular power price. Therefore power peaks, even for a short time, can be extremely costly.

Université Laval has an independent electricity network, and acquires its electrical energy via two $25 \mathrm{kV}$ three phase power lines supplied by Hydro-Québec. Université Laval subscribes to a power of $15.75 \mathrm{MW}$ and maintains its power factor between 0.95 and 1.0 .

This study uses real data provided by the Building Services of Université Laval campus. This data includes instantaneous power consumption for the whole campus every 15 minutes. Consequently each day is divided into 15-minute intervals for the simulation process. We make the assumption that the power drained by the campus remains stable during these intervals. This billing scheme was used in the model to determine the financial efficiency of V2B. Moreover, the campus uses an electric boiler to regulate its power consumption. For our study, we removed the power consumed by the boiler since this element is in direct competition with the use of V2B as it was specifically installed to take advantage of the tariff system through one-way regularization of demand.

The scenario investigated here aims at being beneficial for both parking lot users and the Universite Laval in the following manner: with V2B the power component of the campus bill is reduced and the vehicles are allowed to charge for free in exchange for the right to control the vehicle energy.

\section{OPTIMIZATION}

Optimization is conducted using linear programming, a classical method for convex optimization of a mathematical model expressed as a set of linear equations defining an objective function and constraints. The model maximizes the energy value given to the vehicles while attempting to maintain the overall power consumption at or below a threshold, the subscribed power. The model also considers battery degradation induced by V2B activity. The resulting objective function to be maximized takes into account these concerns and should be read as the overall community benefits of V2B (all in \$):

$$
\begin{aligned}
\max \sum_{t=1}^{T} \sum_{i=1}^{N}[\underbrace{K_{\text {chg }} B 2 V_{i}^{t}}_{\text {charging }}-\underbrace{\left(K_{\text {chg }}+K_{\text {wear }}\right) V 2 B_{i}^{t}}_{\text {discharging }}] \\
-\underbrace{K_{\text {peak }} \gamma^{d}}_{\text {power peak }}-\underbrace{K_{\text {penalty } \gamma^{w}}}_{\text {winter peak penalty }},
\end{aligned}
$$

with the sum computed over time steps $t=1, \ldots, T$ and cars $i=1, \ldots, N$

Decision variables optimized by linear programming allow the following elements to be determined:

- $\quad$ B2 $V_{i}^{t}$ : amount of energy for charging vehicle $i$ at time step $t[\mathrm{kWh}]$;

- $\quad V 2 B_{i}^{t}$ : amount of energy for shaving campus power demand peaks by vehicle $i$ at time step $t$ [kWh].

- $\quad \gamma^{d}$ : maximum power consumption exceeding the subscribed power value $[\mathrm{kW}]$;

- $\quad \gamma^{w}$ : maximum power consumption exceeding $110 \%$ of the original subscribed power in winter $[\mathrm{kW}]$.

The following parameters are given to the model:

- $\quad K_{c h g}$ : charging cost per energy unit $[\$ / \mathrm{kWh}]$;

- $K_{\text {wear }}$ : cost of the battery wear-off induced by discharging a vehicle $[\$ / \mathrm{kWh}]$;

- $K_{\text {peak }}$ : cost of the power consumption exceeding the subscribed power value $[\$ / \mathrm{kW}]$;

- $K_{\text {penalty }}$ : cost of the power consumption exceeding $110 \%$ of the original subscribed power value during the winter $[\$ / \mathrm{kW}]$;

The model also includes a set of constraints that can be interpreted this way:

- $\quad B 2 V_{i}^{t} \in\left[0, w_{i} e_{i}\right]:$ vehicle charging is limited to maximum theoretical energy flow on the grid side for a 15-minute period $\left(w_{i}\right)$ times charger efficiency $\left(e_{i}\right)$;

- $\quad V \mathscr{2} B_{i}^{t} \in\left[0, w_{i} / e_{i}\right]$ : energy from vehicle used for peak shaving is limited to maximum theoretical energy flow on the grid side for a 15-minute period $\left(w_{i}\right)$ divided by charger efficiency $\left(e_{i}\right)$;

- $\quad S o C_{i}^{T}>S o C_{i}^{u n p l u g}$ : SoC when vehicle is disconnected from the charging station should be higher than the minimum charge level requested $\left(S o C_{i}^{u n p l u g}\right)$;

- $S o C_{i}^{t} \in[0.2,0.8]:$ SoC is limited to the $20 \%-80 \%$ range.

The last constraint relies on the fact that the charging and discharging functions are linear and that we can use $60 \%$ of the amplitude of charge of the batteries (20\% to $80 \%$ ). Operating below $20 \%$ would diminish battery life substantially, and so significantly increase wear-off. Over $80 \%$, charging is switched to a constant voltage mode, with current varying over time, resulting in an asymptotically low charging rate, which cannot 
TABLE I. VEHICLES PROPERTIES.

\begin{tabular}{c|c|c}
\hline Property & Prius & Leaf \\
\hline Battery Capacity (kWh) & 4.4 & 24.0 \\
Maximum Charger Intensity (Amps) & $15 \mathrm{AC}$ & $125 \mathrm{DC}$ \\
Maximum Charger Voltage (V) & $240 \mathrm{AC}$ & $480 \mathrm{DC}$ \\
Charger Efficiency (\%) & 93 & 93 \\
\hline
\end{tabular}

TABLE II. SIMULATION PARAMETERS.

\begin{tabular}{c|c|c|c}
\hline Parameter & Minimum & Maximum & \% fleet present \\
\hline Night Time & $0: 00 \mathrm{am}$ & $7: 30 \mathrm{am}$ & $0 \%$ \\
Arrival Time & $7: 30 \mathrm{am}$ & $9: 30 \mathrm{am}$ & stochastic \\
Day Time & $9: 30 \mathrm{am}$ & $3: 30 \mathrm{am}$ & $100 \%$ \\
Departure Time & $3: 30 \mathrm{pm}$ & $5: 30 \mathrm{pm}$ & stochastic \\
Evening Time & $5: 30 \mathrm{pm}$ & $12: 00 \mathrm{pm}$ & $0 \%$ \\
Arrival SoC $(\%)$ & 20 & 80 & \\
\hline
\end{tabular}

be modelled by linear equations. Moreover, charging over $80 \%$ would then be very slow, resulting in negligible gains for the whole system.

\section{Methodology}

The system is built around a scalable number of vehicles which all have their own properties, as shown in Table I, reflecting the specifications of two vehicles available commercially: the Toyota plug-in Prius hybrid 2012 (referred to as "Prius" thereafter) and the Nissan Leaf 2012, which is purely electric. This gives us both level 2 charging (240 V / 15 A AC) and fast charging (480 V / $125 \mathrm{~A} \mathrm{DC}$ ). For this study, the assumption is made that only one type of vehicle is allowed to plug-in: either all Prius or all Leafs.

The optimization process and subsequent simulation of the impact of the decision sequence on our data is run on every day of a given month so that we can produce a monthly bill similar to the one Hydro-Québec would produce with the power consumption profile modified. We determined that most of the days follow a similar pattern both in terms of hourly usage and maximum power peak, whereas a few days in a given month exhibit an unusually high power demand. This effectively means that while we could reduce the power consumption for each day individually, this is not a satisfactory option since we would then increase the battery wear-out for no real gain as the maximum peak for the month is retained for the bill calculation and not the maximum peak of each individual day.

Simulations are repeated with multiple pre-determined parameters in order to compare the outcomes of different possible scenarios:

- Different vehicle fleet sizes: from 100 vehicles to 400 by a step of 100 (4 sizes).

- Different vehicle types: for each scenario, it is assumed that the complete fleet is present on the campus every working day with a progressive arrival and a gradual departure, as described in Table II.

- Different subscribed powers: 16 MW and 16.4 MW.

The simulations take place on a daily basis, for each working day. The arrival SoC value, arrival and departure time of each vehicle are randomly initialized in the ranges given in Table II. The arrival and departure times are determined using
TABLE III. MODEL PARAMETERS.

\begin{tabular}{c|c|c}
\hline Parameter & Summer 2011 & Winter 2011 \\
\hline$K_{\text {peak }}$ & $11.1033 \$ / \mathrm{kW}$ & $11.1027 \$ / \mathrm{kW}$ \\
$K_{\text {penalty }}$ & - & $7.11 \$ / \mathrm{kW}$ \\
$K_{\text {chg }}$ & $0.0242 \$ / \mathrm{kWh}$ & $0.024 \$ / \mathrm{kWh}$ \\
$K_{\text {wear }}$ & $0.2 \$ / \mathrm{kWh}$ & $0.2 \$ / \mathrm{kWh}$ \\
\hline
\end{tabular}

a uniformly distributed random number in the ranges while the arrival state of charge is determined using a triangular distribution centred on $50 \%$. This parametrization aims to reflect the employees' habits, not the students' habits which are expected to be more random.

The minimum battery capacity when unplugging a vehicle, So $C_{i}^{\text {unplug }}$, is determined as:

$$
S o C_{i}^{\text {unplug }}=\max \left(S o C_{i}^{0}, \min \left(0.8,1.1-S o C_{i}^{0}\right)\right),
$$

where $S o C_{i}^{0}$ is the arrival $\mathrm{SoC}$ value. The values generated for $S o C_{i}^{u n p l u g}$ therefore represent $30 \%$ to $80 \%$ SoC for each vehicle in order to prevent them from leaving with less energy than required to make their trip back home.

Additionally the model parameters explained in Section II are given in Table III. $K_{c h g}$ is the typical price for a household in Québec for $1 \mathrm{kWh}$ minus the price for the campus for $1 \mathrm{kWh}$. This represents the gain of charging on the campus compared to doing so at home for the users. $K_{\text {peak }}$ is directly issued from the Hydro-Québec billing model, and is the raw price of power minus a credit for supply at medium or high voltage minus an adjustment for transformation loss. This value of $K_{\text {peak }}$ is then weighted by the number of days in the month divided by 30 . A realistic $K_{\text {wear }}$ was determined using a battery cost of $240 \$ / \mathrm{kWh}$ for standard Li-ion batteries and an expected useful life of 1500 cycles from $100 \%$ to $20 \%$ SoC.

Once the daily simulation run for one setup is completed, a monthly bill is generated combining the new maximum peak component $(\mathrm{kW})$ and the revised energy consumption value $(\mathrm{kWh})$ for the campus. The value of the bill is then compared with the original one.

\section{RESUlts AND ANALYsis}

Results have been generated with the model presented for the months of January and May 2011. Four values are of interest for each simulation setup:

- Maximum power: The largest power peak in the month obtained with the V2B fleet considered. This value is used to calculate the power component of the electricity bill;

- $\Delta$ cumulative energy: The increase in energy consumption on the campus throughout the month due to V2B compared to the case with no vehicle plugged-in;

- $\Delta$ bill campus (\$): The variation of the campus bill (reduced if negative, increased if positive) compared to the case with no vehicle plugged-in. This result includes the campus savings associated with the peak reduction and the additional costs associated with the recharging of the vehicle fleet batteries;

- User benefits: The gain shared by all V2B vehicle owners; this value is calculated combining the value 
of exchanged energy and the battery wear-off. This value is under-estimated because it uses the price of residential electricity as billed by Hydro-Québec for the first $30 \mathrm{kWh}$ consumed, which increases after that level. This is especially the case in most households in Québec in winter.

For the two months selected, the results extracted from the optimization show a cost benefit for the use of V2B in peak shaving mode. The cost benefit will vary depending on the number of vehicles, the subscribed power, and the type of vehicle considered. Table IV shows the results obtained with V2B given the different parameters fed to the system. The results will be discussed for each month, with an analysis of the distinct behaviours observed in each case.

For January 2011, the original cost of electricity for the campus was $545,486 \$$, the original maximum power peak was $16.55 \mathrm{MW}$, and the total consumed energy accounted for $9,661,833 \mathrm{kWh}$. Interestingly, the system optimization leads to advantageous benefits when providing peak compensation in association with an increase in the campus subscribed power. Leaving the subscribed power at $15.75 \mathrm{MW}$ would produce a financial burden in terms of battery wear-off that is not sustainable for the users. The results indicate that a slight increase in the university subscribed power in combination with peak shaving is a better choice, as presented in Table IV for a subscribed power increase to either $16 \mathrm{MW}$ or $16.4 \mathrm{MW}$.

a) Prius in January 2011: In January 2011, aiming for a $16 \mathrm{MW}$ subscribed power does not produce satisfactory results for Prius vehicle owners, due to the size of their battery pack. The battery wear-off associated with the massive discharge of the vehicles is not financially compensated for by charging them. Indeed, the energy storage capability available to the system is not large enough to make it financially appealing for the community. An example of this behaviour is shown with a fleet of 400 Prius in Fig. 1, which indicates that energy flows in both directions 15 days out of 31 days of January 2011. Although less beneficial for the drivers, the Prius has the strongest effect in reducing the campus bill due to the small battery size and, therefore, low energy consumption for a $20 \%$ to $80 \%$ recharge, while still providing a peak demand shaving capability comparable to the Volt.

b) Leaf in January 2011: However, as the fleet size is increased for Leaf vehicles, users gain more from participating, the energy storage constraint being lifted. With $16 \mathrm{MW}$ subscribed power, a 200 Leaf fleet yields the best financial advantages for the campus. Larger fleets of these vehicle will slightly increase the campus bill, while significantly increasing the user benefits. As a matter of fact, when the fleet size increases, the number of $\mathrm{kWh}$ supplied by the campus to the vehicles also increases, thus increasing the campus bill.

c) Increasing the subscribed power with V2B: Interestingly, increasing the university subscribed power from 15.75 MW to $16.4 \mathrm{MW}$ with V2B is still beneficial for the campus, with a more moderate use of the vehicle batteries and less wear-off. Nevertheless, the financial advantage is still greater with $16.0 \mathrm{MW}$ compared to $16.4 \mathrm{MW}$ due to the extra cost associated with the higher subscribed power. The combination of a powerful charger and a large energy
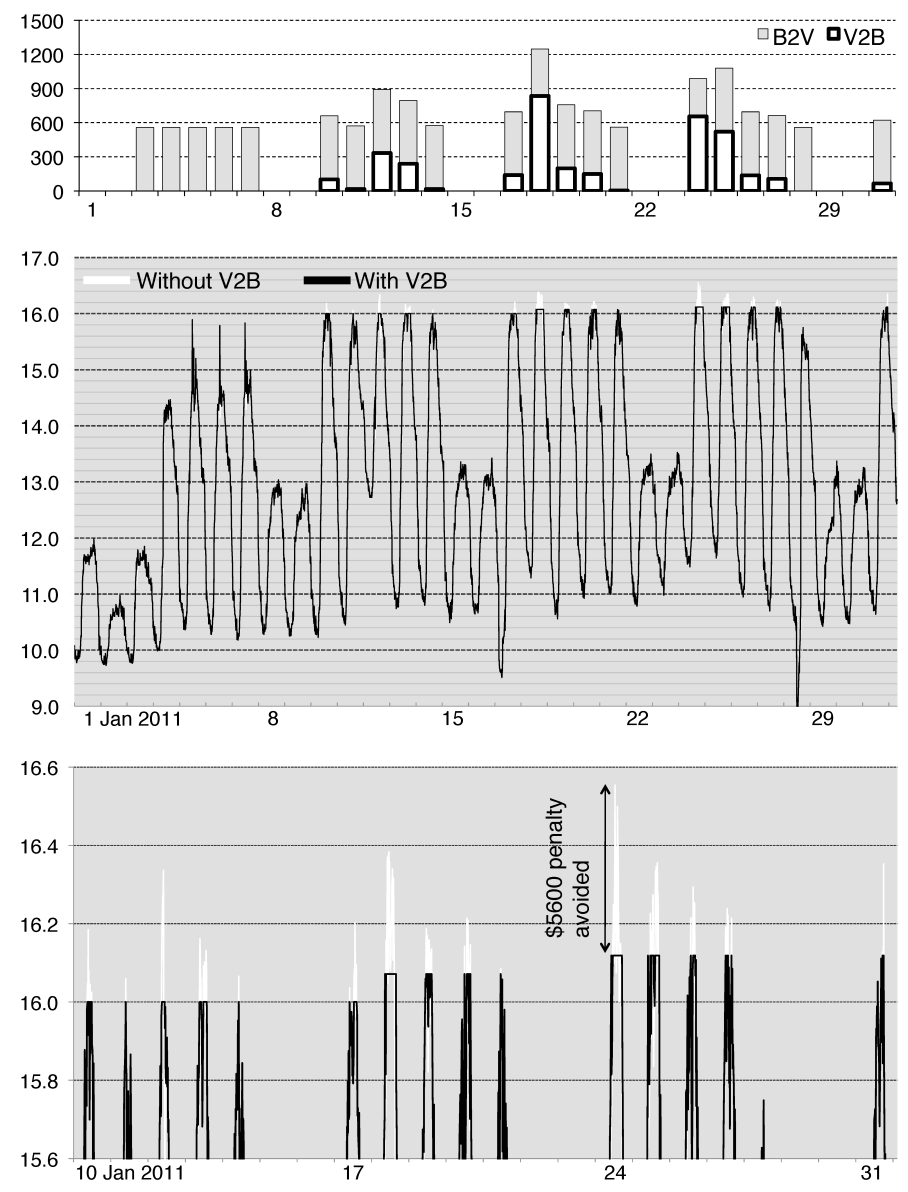

Fig. 1. Power consumption and energy exchanges for the month of January 2011 with 400 Prius and a $16 \mathrm{MW}$ subscribed power. The top figure shows the energy exchanges between the car and the building; the middle figure presents the power calls over the month with and without V2B; and the bottom figure is an excerpt of the power calls figure with the highest peak over the month. Note that in the top figure, the bars are overlapping, both bars starting at zero, the differences between them corresponding to the net energy given to the vehicles.

capacity in the fleet leads to an increased campus bill for 400 Leafs, given that the amount of energy required by the cars is the largest compared to the other tested configurations. It is interesting to note that the maximum power peak achieved with Leafs for a $16 \mathrm{MW}$ goal first decreases with an increasing fleet size, given better capacity to manage the demand, and then increases again, as the larger battery storage requires more energy overall.

\section{d) Adjustment of power demand threshold following} peak days in May 2011: For May 2011, the original cost of electricity for the campus is $534,464 \$$, the original maximum power peak is $16.97 \mathrm{MW}$ and the total consumed energy accounts for $9,239,864 \mathrm{kWh}$. The results for this month are the best yet encountered. Every configuration analyzed is beneficial for both the campus and the users even though the subscribed power is exceeded at $16 \mathrm{MW}$. The graphical results for a balanced case with 400 Leafs and a $16 \mathrm{MW}$ subscribed power is shown in Fig. 2. These results show that, once again, the fleet's major contribution in May 2011 is concentrated on a few days, here the 24th, 30th, and 31st which were in fact the hottest days of the month. The rest of the time, vehicles 

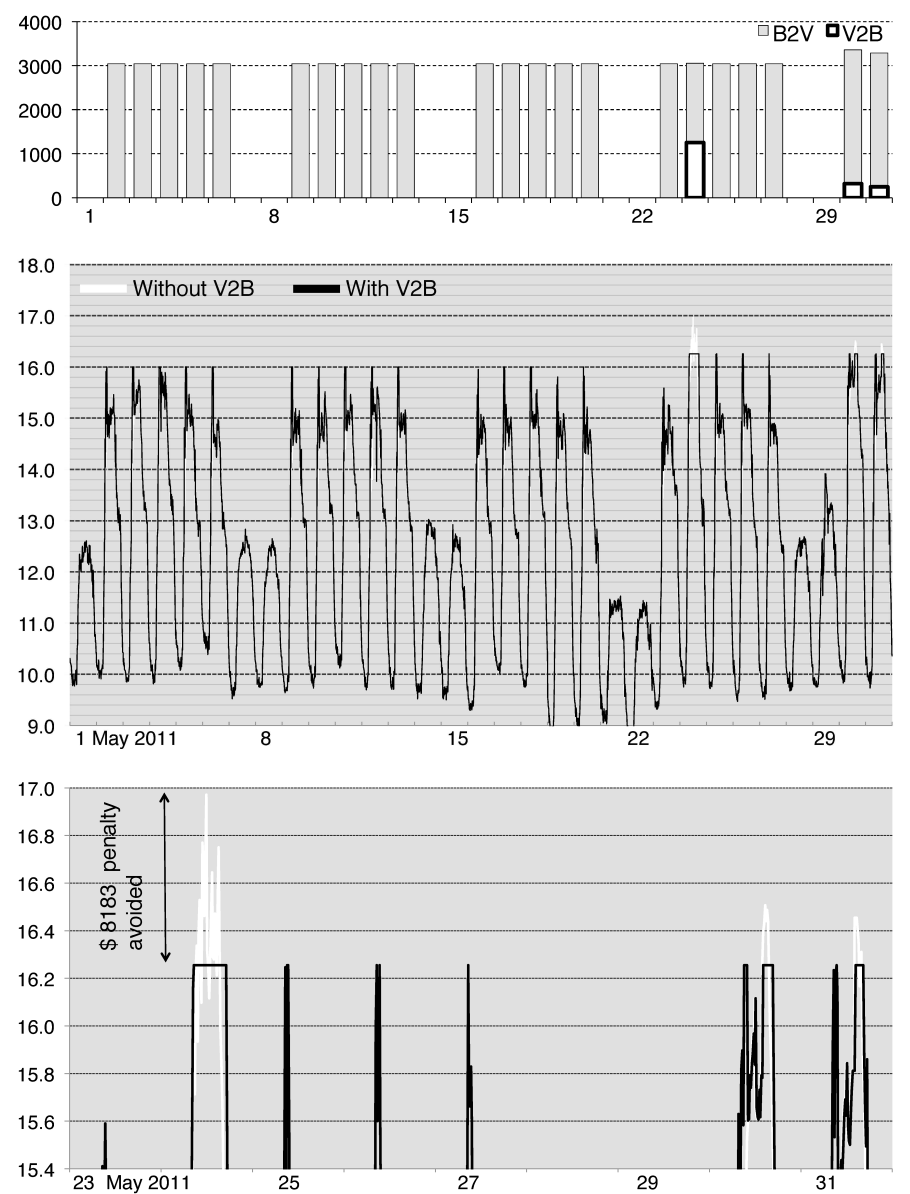

Fig. 2. Power consumption for the month of May 2011 with 400 Leafs and a $16 \mathrm{MW}$ subscribed power. The top figure shows the energy exchanges between the car and the building; the middle figure presents the power calls over the month with and without V2B; and the bottom figure is an excerpt of the power calls figure with the highest peak over the month.

are simply recharged. The effect of the adaptive maximum power goal of the model is best seen in Fig. 2, the peaks never exceeded $16 \mathrm{MW}$ before the 24th. On the 24th, the Leaf fleet could not provide the power needed on this day to remain below the $16 \mathrm{MW}$ threshold. Yet, the power drawn from the utility could be reduced from a previous maximum of 16.97 MW down to $16.26 \mathrm{MW}$. After the 24 th, a new increased threshold of $16.26 \mathrm{MW}$ is used for the remainder of the month, as the $260 \mathrm{~kW}$ power penalty will be billed in any case. This month also exhibits the greatest power peak reduction with $710 \mathrm{~kW}$ fed to the campus by the vehicles.

\section{CONCLUDING REMARKS}

Four main approaches have been identified for smart charging of EVs [3]: uncontrolled charging, off-peak charging, smart charging (valley filling), and smart charging (peak shaving), the latter corresponding to our work. We are making a case with this paper that demand-side management supported by EVs is economically viable in regulated electricity markets. This is relatively novel and different from many studies conducted in the domain, where the emphasis is on deregulated markets, using an electricity price that varies according to supply and demand. In such a setting, unless battery cost is reduced by an order of magnitude, the difference in electricity price over a day is not likely to be at levels allowing peak shaving with EVs for frequent and significant gains. In the context of deregulated markets, V2G would rather be relevant through aggregators [4] that would use a fleet of EVs as a spinning reserve or for frequency regulation [5], [6].

Moreover, the assumption is often made that the V2G infrastructure is already in place at homes and workplaces [7]. This is a strong assumption and we argue that deployment is more likely to happen first in dense parking lots, near office or commercial buildings for instance. Our work in this context is demonstrating that V2B can be economically viable in regulated electricity markets, where the rate is designed to encourage subscribers to ensure that the power call is maintained below certain levels in medium to large organizations. This also present a win-win situation, where EV owners will obtain significant benefits of free charging, with gains even when taking into account battery wear-out, while reducing the building's electricity bill. Such a setting can provide an additional incentive to car owners for making the shift to EVs.

\section{ACKNOWLEDGEMENTS}

This work was supported by funding from the Fonds de Recherche Québécois sur la Nature et les Technologies (FRQNT) and access to computational resources of Calcul Québec/Compute Canada. The authors are grateful to the Buildings Services of Université Laval for providing data used in this project and to Annette Schwerdtfegger for proofreading the paper.

\section{REFERENCES}

[1] W. Kempton, J. Tomić, S. Letendre, A. Brooks, and T. Lipman, "Vehicleto-Grid power: Battery, hybrid, and fuel cell vehicles as resources for distributed electric power in California," UC Davis: Institute of Transportation Studies Report, Tech. Rep. IUCD-ITS-RR 01-03, 2001. [Online]. Available: http://escholarship.org/uc/item/0qp6s4mb

[2] L. Schewel, M. Brylawski, K. Chan-Lizardo, and A. Lovins, "MOVE's smart garage charette report," Rocky Mountain Institute, Tech. Rep. 2008-14, 2008. [Online]. Available: http://www.rmi.org/ Knowledge-Center/Library/2008-14_SmartGarageCharetteReport

[3] J. García-Villalobos, I. Zamora, J. San Martí, F. Asensio, and V. Aperribay, "Plug-in electric vehicles in electric distribution networks: A review of smart charging approaches," Renewable and Sustainable Energy Reviews, vol. 38, pp. 717-731, 2014.

[4] T. G. S. Román, I. Momber, M. R. Abbad, and A. S. Miralles, "Regulatory framework and business models for charging plug-in electric vehicles: Infrastructure, agents, and commercial relationships," Energy Policy, vol. 39, no. 10, pp. 6360-6375, 2011.

[5] W. Kempton and J. Tomić, "Vehicle-to-grid power fundamentals: Calculating capacity and net revenue," Journal of Power Sources, vol. 144, no. 1, pp. 268-279, 2004.

[6] E. Sortomme and M. El-Sharkawi, "Optimal scheduling of Vehicle-toGrid energy and ancillary services," IEEE Transactions on Smart Grid, vol. 3, no. 1, pp. 351-359, 2012.

[7] A. Ashtari, E. Bibeau, S. Shahidinejad, and T. Molinski, "PEV charging profile prediction and analysis based on vehicle usage data," IEEE Transactions on Smart Grid, vol. 3, no. 1, pp. 341-350, 2012. 
TABLE IV. DETAILED RESULTS FOR JANUARY AND MAY 2011 (SP: SUBSCRIBED POWER). BOLD RESULTS CORRESPOND TO THE BEST OPTION GLOBALLY (USER BENEFITS - $\triangle$ BILL CAMPUS) FOR A MONTH, WHILE BOXED RESULTS CORRESPOND TO THE BEST OPTION FOR THE CAMPUS.

\begin{tabular}{|c|c|c|c|c|c|c|c|c|c|}
\hline \multirow[b]{2}{*}{ Car type } & \multirow[b]{2}{*}{ \# } & \multicolumn{2}{|c|}{ Maximum power (MW) } & \multicolumn{2}{|c|}{$\Delta$ cumulative energy $(\mathrm{kWh})$} & \multicolumn{2}{|c|}{$\Delta$ bill campus $(\$)$} & \multicolumn{2}{|c|}{ User benefits (\$) } \\
\hline & & $\mathrm{SP}=16 \mathrm{MW}$ & $\mathrm{SP}=16.4 \mathrm{MW}$ & $\overrightarrow{\mathrm{SP}}=16 \mathrm{MW}$ & $\mathrm{SP}=16.4 \mathrm{MW}$ & $\mathrm{SP}=1 \overline{6} \mathrm{MW}$ & $\mathrm{SP}=16.4 \mathrm{MW}$ & $\mathrm{SP}=16 \mathrm{MW}$ & $\mathrm{SP}=16.4 \mathrm{MW}$ \\
\hline \multicolumn{10}{|c|}{$11=545,486 \$ ;$ power peak $=16.55$} \\
\hline \multirow{4}{*}{ Prius } & 100 & 16.22 & 16.40 & $3,395.35$ & $3,187.1$ & $-4,256.52$ & $-1,906.78$ & -132.49 & 166.4 \\
\hline & 200 & 16.16 & 16.40 & $6,742.06$ & $6,513.52$ & $-4,877.46$ & $-1,793.47$ & -138.84 & 356.35 \\
\hline & 300 & 16.14 & 16.40 & $9,865.27$ & $9,654.69$ & $-5,105.35$ & $-1,686.47$ & -67.39 & 535.72 \\
\hline & 400 & 16.12 & 16.40 & $12,703.7$ & $12,601.82$ & $-5,265.54$ & $-1,586.08$ & -7.14 & 704.0 \\
\hline \multirow{4}{*}{ Leaf } & 100 & 16.08 & 16.40 & $17,542.25$ & $17,335.75$ & $-5,634.9$ & $-1,424.82$ & 47.7 & 974.32 \\
\hline & 200 & 16.03 & 16.40 & $34,764.64$ & $35,479.87$ & $-5,647.22$ & -806.76 & 834.18 & $2,010.38$ \\
\hline & 300 & 16.04 & 16.40 & $50,648.35$ & $52,613.53$ & $-4,987.15$ & -223.11 & $1,790.42$ & $2,988.75$ \\
\hline & 400 & 16.05 & 16.40 & $65,531.5$ & $68,688.78$ & $-4,364.87$ & 324.48 & $2,691.37$ & $3,906.68$ \\
\hline \multicolumn{10}{|c|}{$\mathrm{MW} ; \mathrm{SP}=15.75 \mathrm{MW})$} \\
\hline \multirow{4}{*}{ Prius } & 100 & 16.61 & 16.61 & $3,358.97$ & $3,358.97$ & $-4,591.94$ & $-4,591.94$ & 146.65 & 146.65 \\
\hline & 200 & 16.39 & 16.40 & $6,888.42$ & $6,929.42$ & $-7,350.86$ & $-7,226.4$ & 212.68 & 225.63 \\
\hline & 300 & 16.35 & 16.40 & $10,167.34$ & $10,221.65$ & $-7,808.13$ & $-7,115.01$ & 343.38 & 413.62 \\
\hline & 400 & 16.32 & 16.40 & $13,206.48$ & $13,309.12$ & $-8,011.46$ & $-7,010.54$ & 487.9 & 589.92 \\
\hline \multirow{4}{*}{ Leaf } & 100 & 16.28 & 16.40 & $18,193.94$ & $18,268.47$ & $-8,409.34$ & $-6,842.74$ & 704.05 & 873.11 \\
\hline & 200 & 16.24 & 16.40 & $36,805.59$ & $37,276.6$ & $-8,260.95$ & $-6,199.58$ & $1,700.04$ & $1,958.51$ \\
\hline & 300 & 16.25 & 16.40 & $54,403.73$ & $55,226.15$ & $-7,563.69$ & $-5,592.24$ & $2,719.93$ & $2,983.47$ \\
\hline & 400 & 16.26 & 16.40 & $70,874.48$ & $72,066.88$ & $-6,952.3$ & $-5,022.4$ & $3,667.86$ & $3,945.11$ \\
\hline
\end{tabular}

